\title{
Impacto ambiental de tres estrategias fitosanitarias para el control de Lobesia botrana
}

\author{
Graciela Mendoza ${ }^{\mathrm{a}}$, Jésica Sánchez y Violeta Becerra \\ Estación Experimental Agropecuaria Mendoza, INTA, San Martín 3853, Luján de Cuyo, Mendoza, Argentina
}

\begin{abstract}
Resumen. el propósito del trabajo fue cuantificar, mediante el indicador EIQ el impacto de las estrategias fitosanitarias empleadas para el control de Lobesia botrana en Mendoza. Asimismo, comparar los costos y evaluar la utilidad del indicador. Para ello se analizaron 3 estrategias: A. con clorpirifos; B. con metoxifenocide y clorantraniliprole; C. con Bacillus thuringiensis subsp. kurstaki (Btk) y spinosad. Los resultados obtenidos indican que la estrategia de mayor impacto ambiental es la A, siendo a la vez la de menor costo. La de menor impacto ambiental es la $\mathrm{B}$, mientras que la $\mathrm{C}$ tiene un costo mayor. Por las variables que intervienen en la ecuación, el EIQ obtenido para cada estrategia respondió proporcionalmente a la cantidad de principio activo, dosis utilizadas y número de aplicaciones. Se observó que existen dificultades inherentes al integrar en un único valor, variables tan diversas como la toxicidad crónica al humano, la persistencia y la sistematicidad. Esto limita la utilidad y fiabilidad del modelo. El análisis de costos permite concluir que las diferencias observadas se harán menos significativas, si se ponen en evidencia las externalidades de las distintas estrategias.
\end{abstract}

\section{Introduction}

La vitivinicultura es la principal actividad agroindustrial de la provincia de Mendoza [1,2]. La superficie cultivada con vid es de aproximadamente 160.000 hectáreas (Mendoza concentra el $70 \%$ de la superficie nacional), con el $98,65 \%$ del total implantadas con variedades de vinificar [3]. Debido a la aridez que caracteriza a la región, históricamente se aplicaron cantidades limitadas de agroquímicos, en comparación con otras regiones vitivinícolas del mundo, como las de Europa mediterránea. Los fungicidas, para el control de oidio, peronóspora y podredumbre de los racimos, han sido los principales productos empleados, especialmente cuando las condiciones meteorológicas son favorables para el desarrollo de enfermedades $[4,5]$. Pero la privilegiada situación en que se desarrolló la viticultura mendocina fue afectada a comienzos del siglo XXI, por la cochinilla harinosa de la vid, Planococcus ficus y, a partir del 2010, por la introducción de la polilla de la vid, Lobesia botrana, cuarentenaria para Argentina [6,7].

En particular, la polilla de la vid, ha modificado las prácticas fitosanitarias que el productor vitícola normalmente realizaba al cultivo. Los propietarios y/o responsables técnicos de establecimientos productivos, ubicados en áreas bajo cuarentena, deben elaborar e implementar de manera obligatoria un programa de control químico-biológico, según recomendaciones efectuadas periódicamente por SENASA [8]. Entre los plaguicidas autorizados para su control, la Resolución No 504/2010 incluye aquellos de acción específica, baja toxicidad, pero más costosos y algunos de amplio espectro de acción, mayor toxicidad y menor precio [9]. Estas prácticas

\footnotetext{
${ }^{a}$ Corresponding author: mendoza.graciela@inta.gob.ar
}

pueden tener derivaciones negativas sobre el medio ambiente, la salud y seguridad del trabajador agrícola, la inocuidad de la cosecha y la economía del productor [1015]. Entre las primeras se destacan:

- Disminución de la biodiversidad y eliminación de especies claves (p. ej. abejas).

- Polución del agua.

- Contaminación del suelo.

- Resistencia de plagas, que finalmente resulta en la necesidad de incremento en el número de aplicaciones, o alternancia con plaguicidas de diferente mecanismo de acción.

Estas consecuencias han sido han sido ampliamente estudiadas y reconocidas por distintas instituciones académicas y científicas, y agencias internacionales, como la FAO e IICA. Por ello desde fines del siglo XX colocaron en sus agendas la necesidad de reorientar los sistemas productivos, en la búsqueda de prácticas que garanticen la subsistencia de los recursos naturales, agua, suelo, vegetación y fauna [16-20]. Se considera que una actividad agrícola es ambientalmente sostenible cuando el entorno natural puede soportar en el largo plazo el uso de los recursos naturales y las emisiones contaminantes [21]. Por lo tanto, el diagnóstico del impacto ambiental, constituye el primer paso para la concreción de la sostenibilidad del sistema agrícola. Al ser los plaguicidas uno de los insumos que al ingresar al predio, afectan el logro de la sustentabilidad, a nivel internacional hay una exigencia respecto al desarrollo de herramientas que permitan comparar los efectos ambientales de diferentes productos y métodos de control de plagas en un cultivo [22]. Pero, debido a la complejidad inherente de evaluar el riesgo involucrado y determinar qué práctica de manejo es más inocua, bajo condiciones específicas, el desarrollo de 
herramientas de decisión confiables continúa siendo un desafío para los investigadores [22]. A fin de facilitar el cambio a productos y prácticas de control, que permitan reducir la carga de tóxicos en el medio ambiente, en distintos ámbitos se han desarrollado indicadores de riesgo de plaguicidas, modelos matemáticos y software. Los índices se basan en modelos algebraicos que asignan puntajes a diferentes variables ambientales o indicadores. Tales variables incluyen propiedades físico-químicas de los plaguicidas (p. ej. solubilidad), pruebas de puntos críticos (p. ej. dosis letal $50\left(\mathrm{DL}_{50}\right)$ para un organismo de prueba) y evaluaciones categóricas de un peligro, impacto o riesgo (p. ej. incidencia alta, moderada, baja o ninguna sobre organismos acuáticos). Normalmente son ponderadas por coeficientes que reflejan la importancia relativa, percibida o medida, de la característica para el sistema de evaluación. Los valores se ponderan e integran en ecuaciones matemáticas para crear un índice de evaluación compuesto [22].

El precursor de lo que es, tal vez, el primer modelo de evaluación algebraica del impacto de plaguicidas fue el entomólogo Robert Metcalf. Su objetivo era evaluar qué insecticidas de uso común, empleados a mediados de los '70, eran más adecuados para un Manejo Integrado de Plagas [23]. Bajo la misma premisa básica Kovach et al. (1992), propusieron un modelo algebraico denominado Environmental Impact Quotient (EIQ) o Cociente de Impacto Ambiental, a fin de cuantificar el impacto de los plaguicidas más comunes utilizados en frutales y hortalizas, en la agricultura comercial [24]. Si bien los autores aclaran que comparaciones de toxicidad ambiental de una cantidad dada de ingredientes activos, se puede realizar dentro de una misma clase de plaguicida ( $\mathrm{p}$. ej. insecticidas), ellos comparan, en diferentes estrategias, productos de distinta clase. De esta manera el valor obtenido se puede utilizar para comparar los diferentes plaguicidas y programas de manejo de plagas y determinar, en última instancia, qué programa o plaguicidas tienen, probablemente, el menor impacto ambiental. Este modelo ha sido usado por productores del Estado de Nueva York y a nivel mundial, con fines de marketing y en la toma de decisiones [14,25].

En vista de la actual situación fitosanitaria que aqueja a los viticultores mendocinos, se consideró importante estimar el impacto ambiental de distintas estrategias y analizar la conveniencia del indicador utilizado. Para ello, se decidió realizar una primera aproximación al cálculo del impacto ambiental, mediante la aplicación del índice EIQ, a estrategias usadas para el control de Lobesia botrana. Asimismo analizar los costos de dichas estrategias, ya que en algunos casos el empleo de plaguicidas de bajo impacto ambiental, puede elevar la relación costo/beneficio, en términos netamente económicos. El objetivo final es contar con herramientas confiables, que permitan dar respuesta a las consultas de los productores afectados.

\section{Objetivos}

Calcular y comparar el impacto ambiental de estrategias fitosanitarias empleadas para el control de Lobesia botrana en la viticultura mendocina, mediante el indicador EIQ.
Contrastar los costos directos de cada estrategia. La utilidad del indicador EIQ también será discutida.

\section{Materiales y métodos}

Se diseñaron tres estrategias fitosanitarias para el control de Lobesia botrana, con productos permitidos según Resolución N ${ }^{\circ}$ 504/2010 de SENASA. Los momentos de aplicación corresponden a los alertas de SENASA, que para la primera generación del insecto, en racimo visible, se produjo entre el 17/10/13 y 5/11/13. Para la segunda generación, en cierre de racimo, alrededor del 16/12/13 y la tercera generación, con fruto en envero, a fines de enero de 2014.

Las estrategias diseñadas fueron:

- Estrategia A, considerada "convencional" con cuatro aplicaciones de clorpirifos $75 \%$ WG, en dosis de $80 \mathrm{~g} \mathrm{hl}^{-1}$, dos en $1^{\circ}$ generación y una en cada una de las siguientes generaciones.

- Estrategia B, con productos compatibles con un manejo integrado de plagas: dos aplicaciones de metoxifenocide $24 \% \mathrm{SC}$, en dosis de $30 \mathrm{~cm}^{3} \mathrm{hl}^{-1}$ en $1^{\mathrm{a}}$ generación. En $2^{\mathrm{a}} \mathrm{y} 3^{\mathrm{a}}$ generación una aplicación de clorantraniliprole, $20 \%$ SC, a razón de $20 \mathrm{~g} \mathrm{hl}^{-1}$.

- Estrategia C, con productos biológicos, permitidos en agricultura orgánica: cinco aplicaciones de Bacillus thuringiensis subsp. kurstaki (Btk) 3,5 \% EC, de $1,5 \mathrm{~L} \mathrm{hl}^{-1}$, y una de spinosad al $48 \% \mathrm{SC}$, de $20 \mathrm{~cm}^{3} \mathrm{hl}^{-1}$.

El volumen empleado, en la $1^{\text {a }}$ generación del insecto, fue de $40 \mathrm{~L} \mathrm{ha}^{-1}$, debido a la menor cobertura de follaje, mientras que en $2^{\mathrm{a}}$ y $3^{\mathrm{a}}$ generación fue de $800 \mathrm{~L} \mathrm{ha}^{-1}$.

Para la evaluación del impacto ambiental se empleó el EIQ, cuya fórmula consta de tres componentes: toxicidad al trabajador agrícola, al consumidor y a la biota no humana. Como resultado de promediar los tres componentes se obtiene un único valor de EIQ, según el siguiente modelo:

$\mathrm{EIQ}=1 / 3$ (EIQ trabaj. agríc. + EIQ consum. + EIQ ecol.)

Donde:

EIQ trabajador agrícola: C (DT x 5) + (DT x P)

EIQ consumidor: $\mathrm{C}[(\mathrm{S}+\mathrm{P}) / 2] \mathrm{SY}+\mathrm{L}$

EIQ ecológico: $(\mathrm{F} \times \mathrm{R})+\{\mathrm{D}[(\mathrm{S}+\mathrm{P}) / 2] \times 3\}+(\mathrm{Z} \times \mathrm{P} \times$ $3)+(\mathrm{B} \times \mathrm{P} \times 5)$.

En la fórmula (1) cada componente está formado por variables cuyos datos de entrada han sido transformadas en calificaciones numéricas o categorías de peligro en una escala arbitraria de 1 a 5, según el criterio establecido por Kovach et al. (1992), que se describe en la Tabla 1 [24]. Además algunas variables de la fórmula se ponderan. En el impacto al trabajador agrícola, la toxicidad dermal aguda se pondera por un factor de 5 , debido a la mayor probabilidad de exposición del aplicador al producto concentrado. En el componente del consumidor, en cambio, las variables no se ponderan. Por otro lado, en el componente ecológico, la toxicidad a las aves y abejas se pondera por 3, para asignar mayor probabilidad de exposición de los organismos terrestres con respecto a 
Tabla 1. Categorías numéricas de las variables asignadas por Kovach et al. (1992).

\begin{tabular}{|c|c|c|c|c|}
\hline & & \multicolumn{3}{|c|}{ Puntajes y criterios } \\
\hline Variable & Sigla & 1 & 3 & 5 \\
\hline $\begin{array}{l}\text { Toxic. } \\
\text { crónica }\end{array}$ & $\mathrm{C}$ & Baja o nula & Posible & Definitiva \\
\hline $\begin{array}{l}\text { Toxic. } \\
\text { dermal } \\
\text { aguda } \\
\text { (DL }{ }_{50} \text { dermal) }\end{array}$ & DT & $2000 \mathrm{ppm}$ & $\begin{array}{l}200- \\
2000 \mathrm{ppm}\end{array}$ & $\begin{array}{l}0- \\
200 \mathrm{ppm}\end{array}$ \\
\hline $\begin{array}{l}\text { Toxicidad } \\
\text { pájaros } \\
\text { (CL50 ocho } \\
\text { días) }\end{array}$ & $\mathrm{D}$ & $\begin{array}{l}> \\
1000 \mathrm{ppm}\end{array}$ & $\begin{array}{l}100- \\
1000 \mathrm{ppm}\end{array}$ & $\begin{array}{l}1- \\
100 \mathrm{ppm}\end{array}$ \\
\hline $\begin{array}{l}\text { Letalidad } \\
\text { para abejas } \\
\text { (dosis a } \\
\text { campo) }\end{array}$ & $\mathrm{Z}$ & $\begin{array}{l}\text { Relativa- } \\
\text { mente no } \\
\text { tóxico. }\end{array}$ & $\begin{array}{l}\text { Modera- } \\
\text { mente } \\
\text { tóxico }\end{array}$ & $\begin{array}{l}\text { Altamente } \\
\text { tóxico }\end{array}$ \\
\hline $\begin{array}{l}\text { Toxic. } \\
\text { artrópodos } \\
\text { benéficos }\end{array}$ & B & $\begin{array}{l}\text { Impacto } \\
\text { bajo }\end{array}$ & $\begin{array}{l}\text { Impacto } \\
\text { moder- } \\
\text { ado }\end{array}$ & $\begin{array}{l}\text { Impacto } \\
\text { severo }\end{array}$ \\
\hline $\begin{array}{l}\text { Toxic. peces } \\
\left(\mathrm{CL}_{50} 96 \mathrm{~h}\right)\end{array}$ & $\mathrm{F}$ & $>10 \mathrm{ppm}$ & $1-10 \mathrm{ppm}$ & $<1 \mathrm{ppm}$ \\
\hline $\begin{array}{l}\text { Vida media } \\
\text { en suelo }\end{array}$ & $\mathrm{S}$ & $<30$ días & $\begin{array}{l}\text { 30-100 } \\
\text { días }\end{array}$ & $>100$ días \\
\hline $\begin{array}{lr}\text { Vida } & \text { media } \\
\text { en } & \text { sup. } \\
\text { planta } & \end{array}$ & $\mathrm{P}$ & $\begin{array}{l}1-2 \\
\text { semanas }\end{array}$ & $\begin{array}{l}2-4 \text { sem- } \\
\text { anas }\end{array}$ & $\begin{array}{l}4 \\
\text { semanas }\end{array}$ \\
\hline Sistematic. & SY & $\begin{array}{l}\text { no } \\
\text { sistémico }\end{array}$ & sistémico & \\
\hline $\begin{array}{l}\text { Lixiviación } \\
\text { potencial* }\end{array}$ & $\mathrm{L}$ & poca & media & $\begin{array}{l}\text { Impor- } \\
\text { tante }\end{array}$ \\
\hline $\begin{array}{l}\text { Escorrent. } \\
\text { Potencial * }\end{array}$ & $\mathrm{R}$ & poca & media & $\begin{array}{l}\text { Impor- } \\
\text { tante }\end{array}$ \\
\hline
\end{tabular}

* Tienen en cuenta: vida media en el agua, solubilidad, coeficiente de adsorción y propiedades del suelo.

Tabla 2. EIQ de los distintos componentes de la fórmula EIQ, para los plaguicidas empleados en el control de Lobesia botrana.

\begin{tabular}{|l|l|l|l|l|}
\hline $\begin{array}{l}\text { Princip. ac- } \\
\text { tivo }\end{array}$ & $\begin{array}{l}\text { EIQ } \\
\text { trab. } \\
\text { agríc. }\end{array}$ & $\begin{array}{l}\text { EIQ } \\
\text { con- } \\
\text { sum. }\end{array}$ & $\begin{array}{l}\text { EIQ } \\
\text { ecológ. }\end{array}$ & $\begin{array}{l}\text { EIQ } \\
\text { total }\end{array}$ \\
\hline Clorpirifos & 6 & 2 & 72,55 & 26,85 \\
\hline Metoxifenoc. & 10 & 8 & 78,25 & 32,8 \\
\hline Clorantranil. & 6,9 & 6,45 & 41,66 & 18,34 \\
\hline Btk & 6,9 & 2,45 & 30,63 & 13,3 \\
\hline Spinosad & 6 & 2 & 35,15 & 14,38 \\
\hline
\end{tabular}

los acuáticos. Para los artrópodos benéficos se pondera por 5, considerando que pasan más tiempo en el cultivo.

Los valores de EIQ para cada plaguicida analizado en este trabajo, son los publicados en la página web Integrated Pest Management Program de la Universidad de Cornell, según se muestra en la Tabla 2 [26].

El impacto ambiental de cada plaguicida se obtiene, según la metodología propuesta por Kovach et al. (1992), como el producto entre el EIQ correspondiente, el porcentaje de principio activo (p.a.) de la formulación aplicada, la dosis en $\mathrm{kg}$ o $\mathrm{Lha}^{-1}$ y el número de aplicaciones realizadas. Luego el EIQ de cada estrategia es la suma de los EIQ obtenidos para cada plaguicida.
Tabla 3. EIQ para la estrategia A.

\begin{tabular}{|l|l|l|l|l|l|l|}
\hline p.a. & EIQ & $\begin{array}{l}\text { \% p.a. } \\
\text { for- } \\
\text { mul. }\end{array}$ & dosis & $\begin{array}{l}\mathrm{N}^{\text {o }} \\
\text { de } \\
\text { apli- } \\
\text { cac. }\end{array}$ & $\begin{array}{l}\text { EIQ } \\
\text { par- } \\
\text { cial }\end{array}$ & $\begin{array}{l}\text { EIQ } \\
\text { final }\end{array}$ \\
\hline Clorpir. & 26,85 & 0,75 & 0,32 & 2 & 12,89 & \multirow{2}{*}{38,66} \\
\hline Clorpir. & 26,85 & 0,75 & 0,64 & 2 & 25,78 & \\
\hline
\end{tabular}

Tabla 4. EIQ para la estrategia B.

\begin{tabular}{|l|l|l|l|l|l|l|}
\hline p.a. & EIQ & $\begin{array}{l}\% \\
\text { p.a. } \\
\text { for- } \\
\text { mul. }\end{array}$ & dosis & $\begin{array}{l}\text { N }^{\circ} \text { de } \\
\text { aplicad }\end{array}$ & EIQ parc. & $\begin{array}{l}\text { EIQ } \\
\text { final }\end{array}$ \\
\cline { 1 - 5 } Metoxif. & 32,8 & 0,24 & 0,12 & 2 & 1,89 & \multirow{2}{*}{3,06} \\
\hline Clorantr. & 18,34 & 0,2 & 0,16 & 2 & 1,17 & \multirow{2}{|c}{} \\
\hline
\end{tabular}

Tabla 5. EIQ para la estrategia $C$

\begin{tabular}{|l|l|l|l|l|l|l|}
\hline p.a. & EIQ & $\begin{array}{l}\text { p.a. } \\
\text { for- } \\
\text { mul. }\end{array}$ & dosis & $\begin{array}{l}\mathrm{N}^{\text {o }} \\
\text { de } \\
\text { apli- } \\
\text { cac. }\end{array}$ & $\begin{array}{l}\text { EIQ } \\
\text { parc. }\end{array}$ & $\begin{array}{l}\text { EIQ } \\
\text { final }\end{array}$ \\
\hline Btk & 13,3 & 0,035 & 1,5 & 5 & 3,49 & \multirow{2}{*}{4,32} \\
\hline spinosad & 14,38 & 0,48 & 0,12 & 1 & 0,83 & \\
\hline
\end{tabular}

\section{Resultados y discusión}

\subsection{Cálculo del EIQ}

Los resultados obtenidos de EIQ para las diferentes estrategias que puede implementar el productor vitícola, para el control de Lobesia botrana fueron:

\subsubsection{Análisis de resultados}

Se observa que la estrategia $\mathrm{A}$ es la de mayor impacto ambiental, con un EIQ de 38,66. Dado que el valor inicial de EIQ para el clorpirifos $(26,85)$ es inferior al del metoxifenocide $(32,8)$, la magnitud trece veces superior de la estrategia A respecto a la estrategia B $(3,06)$, está dada por el mayor número de aplicaciones, mayor porcentaje de principio activo en la formulación y mayor dosis utilizada. Asimismo, la estrategia C, con un EIQ de 4,32, es de mayor impacto ambiental que la estrategia B. Esto responde a la elevada dosis de Btk y, dada su escasa persistencia, al número de aplicaciones necesarias para cubrir las 3 generaciones de lobesia. Sin embargo, los datos toxicológicos, muestran que no supone una amenaza para mamíferos ni otros organismos no blanco [27].

Los resultados obtenidos con la metodología EIQ resultan discutibles, al menos los siguientes aspectos:

- La clasificación de toxicidad obtenida, a partir de los valores EIQ para los plaguicidas empleados, no es consistente con las propiedades toxicológicas de los mismos. Al no considerar la dosis letal $50\left(\mathrm{DL}_{50}\right)$ oral aguda, la concentración letal $50\left(\mathrm{CL}_{50}\right)$ inhalatoria, ni ponderar la toxicidad crónica, un compuesto de 
efectos conocidos en seres humanos, no es un riesgo importante para los trabajadores agrícolas a menos que también muestre efectos dérmicos agudos en los ensayos con animales [25]. Se obtiene así una clasificación discutible, si se tienen en cuenta las propiedades citadas, dadas por su estructura química y mecanismo de acción. Así, por ejemplo, el clorpirifos, organofosforado, neurotóxico, tiene una $\mathrm{DL}_{50}$ oral aguda para mamíferos de 135 a $163 \mathrm{mg} \mathrm{kg}^{-1}$ y una $\mathrm{CL}_{50}$ inhalatoria $\left(4 \mathrm{a} 6 \mathrm{~h}\right.$ ) mayor a $0,2 \mathrm{mgL}^{-1} \mathrm{de}$ aire. El metoxifenocide, en cambio, es un regulador del crecimiento de insectos, con una $\mathrm{DL}_{50}$ oral aguda para mamíferos mayor a $5000 \mathrm{mg} \mathrm{kg}^{-1}$ y una $\mathrm{CL}_{50}$ inhalatoria (4 a $6 \mathrm{~h}$ ) mayor a $4,3 \mathrm{mg} \mathrm{L}^{-1}$ de aire [28].

- Debido a las variables que intervienen en la fórmula, los EIQ de metoxifenocide, clorantraniliprole y Btk para los componentes cosechador, trabajador agrícola y consumidor son más elevados que para el clorpirifos.

- En el componente trabajador agrícola, supone de igual importancia la toxicidad crónica y la persistencia. Así para el metoxifenocide la persistencia toma un valor de 5 , porque su vida media foliar es superior a 4 semanas. El clorpirifos, en cambio, se disipa rápidamente, por lo que se le asigna un factor de 1 [29]. Sin embargo, aunque los organofosforados son menos persistentes, potencialmente son más tóxicos para los agricultores y trabajadores del campo, sobre todo si son mal usados o se aplican en condiciones precarias de seguridad, como suele ocurrir en muchos países en vías de desarrollo [30].

- Dado que aún para el producto menos tóxico el menor valor asignable en la escala es 1 , no es posible distinguir entre un producto inocuo y uno de baja toxicidad.

- Tanto en la toxicidad a abejas como a artrópodos benéficos, la persistencia se pondera por diferentes coeficientes, elevando el EIQ del componente ecológico aunque la toxicidad del plaguicida sea muy baja. Debido a ello, existe un desequilibrio respecto a la importancia relativa asignada al componente ecológico.

- Otra limitante son las bases de datos incompletas sobre los efectos toxicológicos y ecológicos de los plaguicidas [22]. Además, hay datos muy limitados sobre los impactos de los nuevos bioplaguicidas, como los fúngicos y microbianos. Kovach et al. (1992) sugieren llenar las lagunas de datos con puntuaciones medias de clases similares de productos químicos, en un rango tan amplio como herbicidas, fungicidas, insecticidas, etc., sin tener en cuenta los diferentes mecanismos de acción dentro de un mismo grupo, o las posibles interacciones entre plaguicidas y los ingredientes inertes presentes en diferentes formulaciones [25].

- Las curvas de degradación de plaguicidas muestran que la relación dosis/respuesta raramente es lineal, por lo que no es correcto asumir que el daño provocado por una estrategia fitosanitaria pueda obtenerse a partir de la suma de los efectos de los plaguicidas individuales [25].

- Un único número ignora el hecho que los efectos medioambientales de un plaguicida dependen de las
Tabla 6. Costos directos de la estrategia A.

\begin{tabular}{|l|l|l|l|l|l|}
\hline p.a. & dosis & $\begin{array}{l}\mathrm{N}^{\text {o de }} \\
\text { aplicac. }\end{array}$ & $\begin{array}{l}\text { costo } \\
\text { unitario } \\
\left(\mathrm{USD} \mathrm{kg}^{-1}\right)\end{array}$ & $\begin{array}{l}\text { costo } \\
\text { apli- } \\
\text { cac. } \\
\text { (USD) }\end{array}$ & $\begin{array}{l}\text { costo total } \\
\left(\mathrm{USD} \mathrm{ha}^{-1}\right)\end{array}$ \\
\hline \multirow{2}{*}{ clorpirifos } & 0,32 & 2 & \multirow{2}{*}{29,24} & 45,91 & 110,5 \\
\cline { 2 - 3 } \cline { 5 - 6 } & 0,64 & 2 & & & 24,3 \\
\hline & & & & & \\
\hline
\end{tabular}

Tabla 7. Costos directos de la estrategia B.

\begin{tabular}{|l|l|l|l|l|l|}
\hline p.a. & dosis & $\begin{array}{l}\mathrm{N}^{\mathrm{o}} \text { de } \\
\text { aplicac. }\end{array}$ & $\begin{array}{l}\text { costo } \\
\text { unitario } \\
\left(\mathrm{USD} \mathrm{kg}^{-1}\right)\end{array}$ & $\begin{array}{l}\text { costo } \\
\text { aplicac. } \\
\text { (USD) }\end{array}$ & $\begin{array}{l}\text { costo total } \\
\left(\mathrm{USD} \mathrm{ha}^{-1}\right)\end{array}$ \\
\hline Metoxif. & 0,12 & 2 & 49,6 & \multirow{2}{*}{45,91} & 103,7 \\
\hline Clorant. & 0,16 & 2 & 396,5 & & 218,7 \\
\hline & & & & & 322,4 \\
\hline
\end{tabular}

Tabla 8. Costos directos de la estrategia C.

\begin{tabular}{|c|c|c|c|c|c|}
\hline p.a. & dosis & $\begin{array}{l}\mathrm{N}^{\circ} \text { de } \\
\text { aplicac. }\end{array}$ & $\begin{array}{l}\text { costo } \\
\text { unitario } \\
\left(\mathrm{USD} \mathrm{kg}^{-1}\right)\end{array}$ & $\begin{array}{l}\text { costo } \\
\text { aplicac. } \\
\text { (USD) }\end{array}$ & $\begin{array}{l}\text { costo total } \\
\left(\text { USD ha }^{-1}\right)\end{array}$ \\
\hline Btk & 1,5 & 5 & 310 & \multirow{2}{*}{370,5} & 517,6 \\
\hline spinosad & 0,12 & 1 & 3390 & & 96,3 \\
\hline & & & & & 614 \\
\hline
\end{tabular}

condiciones en las que es usado. Así, por ejemplo, la contaminación del agua superficial o subterránea, no depende sólo del potencial de escorrentía o de lixiviación del producto sino que puede tener distinta probabilidad de ocurrencia según la distancia que éstas se encuentren de la zona de aplicación [25].

- Otro problema, reside en que se dan valores cuantitativos a medidas cualitativas, por lo que no tienen un significado cuantitativo real [25].

No cabe duda que para comparar cuantitativamente los plaguicidas es necesario tener en cuenta su naturaleza química, interacciones, modo y mecanismo de acción, entre otras variables que hacen al impacto ambiental de estos compuestos.

\subsection{Cálculo de costos}

Se consideraron los valores de productos y de la aplicación, al mes de mayo de 2014.

\subsubsection{Análisis de costos}

Se observa que la estrategia B, de menor EIQ, tiene un costo directo de USD 322,4, que supera en un $34 \%$ al de la estrategia A, de USD 240. La estrategia C, con productos orgánicos tiene un costo de USD 614, que representa casi el doble de la estrategia $\mathrm{B}$, sobre todo por el número de aplicaciones necesarias de Btk.

Al analizar los costos de las distintas estrategias (tablas $\mathrm{N}^{\mathrm{o}} 6,7$ y 8), es posible comprender la preocupación de investigadores, respecto a la posibilidad económica de los 
viñateros mendocinos, de utilizar un plan de control que sea ambientalmente sustentable.

\section{Conclusiones}

Al aplicar el índice EIQ se observa que el orden de clasificación obtenido para las distintas estrategias depende, en parte, de los componentes del análisis. Así se detectan complejidades inherentes al derivar un único valor de índice compuesto y problemas con la estructura matemática (variables evaluadas, datos utilizados, ponderación relativa, etc.), que limitan la utilidad y fiabilidad de este modelo algebraico, para evaluar el impacto de plaguicidas. En concordancia con lo expuesto por Dushoff (1994) y Levitan (1997), en general, estos índices suelen ser poco adecuados para evaluaciones que pretenden reflejar las condiciones específicas de una situación y ser sensibles a tal variabilidad.

Sin embargo, en esta primera aproximación se pone en evidencia que la estrategia con clorpirifos tiene un alto impacto ambiental. Asimismo el menor valor de EIQ y el costo obtenido para la estrategia $\mathrm{B}$, coinciden con las investigaciones que reconocen al MIP como pilar del desarrollo sostenible de la producción agrícola y la reducción del riesgo de plaguicidas. Con respecto a la estrategia $\mathrm{C}$ se destaca que el productor podría basar su decisión en el costo de la misma y no en un análisis del impacto ambiental. Será, entonces, un compromiso de los técnicos hacer evidentes los costos ocultos y externalidades de distintas estrategias de control de plagas, así como diseñar alternativas de menor impacto ambiental, para que puedan ser implementadas por productores de diferentes estratos económicos. Considerar que la aplicación de una estrategia con plaguicidas de amplio espectro de acción, puede producir a largo plazo, la pérdida de enemigos naturales o la resistencia de las plagas, hasta transformarla en económicamente inviable. De esta forma las diferencias de costo se harán menos significativas y será probable que los productores tomen decisiones que les permitan alcanzar la sustentabilidad en todas sus dimensiones.

En vista de resultados obtenidos con la metodología EIQ, queda claro que es necesario profundizar en el desarrollo de una metodología de análisis que contemple todas las variables involucradas, teniendo en cuenta las diferencias propias entre sistemas de cultivo y entre regiones.

\section{Referencias}

[1] C. Catania, et al. El Terruño. Curso superior de degustación. EEA Mendoza INTA. 5-10. (2007). [Disponible en línea]. www.inta.gob.ar/ documentos/curso-de-degustacion-de-vinos/ .../16.\%20E]\%20Terruño.pdf [acceso 20/ 08/2013]

[2] C. Abihaggle, et al. Impacto de la vitivinicultura en la economía argentina 2010. Facultad de Ciencias Económicas y Fondo Vitivinícola. 144 p. (2011). [Disponible en línea]. http://www.fce.uncu. edu.ar/upload/investigacionvitiv.pdf [acceso 08/03/2014]

[3] INV (Instituto Nacional de Vitivinicultura). Distribución por provincias de origen (2012). [Disponible en línea]. http://www.inv.gov.ar/ PDF/Estadisticas/Exportaciones/2012/ ProvinciaOrigenEnero2012.pdf

[4] M. García. Sanidad de la vid. Plagas, virosis, enfermedades y malezas. Ediciones INTA Centro Regional Cuyo. Argentina. 24 p. (1994)

[5] V. Becerra. Residuos de plaguicidas en el cultivo de la vid. Idia XXI. Año I. N ${ }^{0} 1$. Ediciones INTA. 57-59. (2001)

[6] V. Becerra, et al. Dinámica poblacional de Planococcus ficus Sign. (Hemiptera - Pseudococcidae) en viñedos. Mendoza, Argentina. Rev. FCA UNCuyo. Tomo XXXVIII. N 1. 1-6. (2006)

[7] ISCAMEN. Lobesia botrana, lo que hay que saber. Revista Campo Andino. 2 (7). 20 - 22. (2010)

[8] SENASA. Resolución 122/2010. Se declara en todo el territorio de la República Argentina la emergencia fitosanitaria respecto de la plaga Lobesia botrana. [Disponible en línea]. http: //www . senasa.gov . ar/contenido.php? to=n\&in=1447\&io=12300 [acceso 27/05/2013]

[9] SENASA. Resolución 504/2010. Registro Nacional de Terapéutica Vegetal. Autorízanse formulaciones de productos para el control de Lobesia botrana. [Disponible en línea]. http: //www . senasa.gov . ar/contenido.php? to $=$ n\&in=1447\& io=13505 [acceso 20/07/2013]

[10] L. Albert. Plaguicidas, salud y ambiente. Centro Panamericano de Ecología Humana y Salud e Instituto Nacional de Investigaciones sobre recursos bióticos, Metepec, México (1986)

[11] FAO (Food and Agriculture Organization). Lucha Contra la Contaminación Agrícola de los Recursos Hídricos. Estudio riego y drenaje. (1997). [Disponible en línea]. http://www.fao. org/docrep/w2598s/w2598s00.htm\#Contents [acceso 20/06/2014]

[12] Pérez Moreno, I. Fundamentos teóricos del manejo integrado de plagas. Bol. S.E.A. $\mathrm{N}^{\circ} 27,127-133$. (2000)

[13] C. Wilson, et al. Why farmers continue to use pesticides despite environmental, health and sustainability costs. Ecological Economics. 39: 44946. (2001)

[14] I. Macharia, et al. Potential environmental impacts of pesticides use in the vegetable sub-sector in Kenya. Afr. J. Hort. Sci. 2:138-151. (2009). [Disponible en línea]. http://hakenya.net/ajhs/index . $\mathrm{php} / \mathrm{ajhs} /$ article [acceso 12/06/14]

[15] A. Tonolli. Ecología Agrícola y Protección ambiental-XI Agricultura sustentable. Asignatura Ecología Agrícola y Protección Ambiental. Fac. de Ciencias Agrarias, Universidad Nacional de Cuyo. 2011

[16] M. A. Altieri. Manejo y diseño de sistemas agrícolas sustentables. Ministerio de Agricultura, pesca y alimentación. Secretaría Gral. de Estructura 
Agrarias. Madrid. Hoja Divulgadora No 6-7. 52 p. (1994)

[17] D. Tilman, et al. Agricultural sustainability and intensive production practices. Nature. 418. 671 677 (2002)

[18] J. Pretty. Agricultural sustainability: Concepts, principles and evidence. Philosophical Transactions Of The Royal Society B: Biological Sciences, 363, 1491, p. 447 - 465. (2008). Scopus R, EBSCOhost

[19] M. Chiappe. Sustentabilidad de la agricultura: un enfoque integrador. El campo uruguayo: Una mirada desde la sociología rural. Montevideo, Facultad de Agronomía. 251 - 268. (2008)

[20] L. García-Barrios, et. al. Neotropical forest conservation, agricultural intensification, and rural out-migration: the Mexican experience. BioScience 59:863 - 873. (2009)

[21] S. Payraideau, H. Van der Werf. Environmental Impact assessment for a farming region: a review of methods. Agricultural, Ecosystems and Environment 107, 1-19. (2005)

[22] L. Levitan, et al. An Overview of Pesticide Impact Assessement Systems (a.k.a. "Pesticide Risk Indicators"). Based on Indexing or Ranking Pesticides by Environmental Impact. Cornell University. Ithaca, New York, USA. 14853 (1997)

[23] R. L. Metcalf. Insecticides in pest management in R. L. Metcalf and W. H. Luckman, eds., Introduction to insect pest management. New York. 2: 217-277 (1982)
[24] J. Kovach, et al. A Method to Measure the Environmental Impact of Pesticides. New Yorks Life and Sciences Bulletin. Number 139. (1992). [Disponible en línea]. http://dspace. library. cornell.edu/ bitstream/1813/5203/1/FLS-139.pdf [acceso $12 / 06 / 14]$

[25] J. Dushoff, et al. Evaluating the environmental effect of pesticides: a critique to the Environmental Impact Quotient. American Entomology. 180-184. 1994

[26] Cornell University. A Method to measure the environmental impact of pesticide. Table 2: List of Pesticides, Part 4: Insecticides (2012). [Disponible en línea]. http://www.nysipm. cornell. edu/publications/eiq/files/EIQ_values_ 2012insect.pdf [acceso 02/05/2014]

[27] EXTOXTET. Bacillus thuringiensis [en línea; publication date: 5/94]. http://pmep.cce. cornell.edu/profiles/extoxnet/24dcaptan/bt-ext.html [acceso 02/05/2014].

[28] BCPC (British Crop Production Council). The Pesticide Manual. Fifteenth Edition. UK. (2009)

[29] EXTOXTET. Chlorpyrifos [en línea; publication date: 9/93]. http://pmep.cce.cornell.edu/ profiles/extoxnet/carbaryl-dicrotophos/ chlorpyrifos-ext.html [acceso 02/05/2014]

[30] Wilson C., et al. Why farmers continue to use pesticides despite environmental, health and sustainability costs. Ecological Economics 39- 44946. (2001) 